\title{
Preface: Joint Discussion JD7 Space-time reference systems for future research
}

The Joint Discussion on Space-time reference systems for future research (JD7) was held at the XXVIIIth General Assembly of the IAU in Beijing, on 27-29 August 2012. It was organized by IAU Division I (Fundamental Astronomy), with the support of Division III (Planetary Systems Sciences), Division IX (Optical \& Infrared Techniques), Division XI (Space \& High Energy Astrophysics), and Division XII (Union-Wide Activities). The scientific organizing committee was composed of Nicole Capitaine (France; co-Chair), George H. Kaplan (USA), Sergei Klioner (Germany; co-Chair), Zoran Knezevic (Republic of Serbia), Dafydd Wyn Evans (UK), Dennis McCarthy (USA; co-Chair), Harald Schuh (Austria), Richard N. Manchester (Australia) and Gérard Petit (France).

The goal of this Joint Discussion was to coordinate ongoing efforts in the field of the reference frames, models and algorithms used by the international astronomical and space sciences community and to develop effective strategies for future cooperative work to ensure that the reference systems will be sufficiently accurate to meet the future needs. JD7 therefore reviewed currently planned projects devoted to improving reference systems and the links between the various reference frames.

Future astronomical, astrophysical and geophysical research will demand reference systems of the highest accuracy. Past efforts in the development of reference systems have already enabled unprecedented advances in these areas. These advances have, in turn, spurred new investigations that demand even higher accuracy reference systems. Benefits of recent improvements include the determination of the masses of planets from pulsar timings, determination of PPN parameters, accurate stellar distances, and dynamics of the Earth's motion. The validity of the most promising investigations can be limited by the reference frames, models and algorithms used to make the required observations and to analyze the results. Investigations of exoplanets and near-Earth objects demand highly precise reference frames. Future space missions and pulsar timing projects will require the development of relativistic time transfer algorithms and highly accurate planetary ephemerides. Investigations of terrestrial phenomena such as the rise of ocean levels require the most precise reference frames to enable the required astro-geodetic measurements.

The scientific programme of the meeting included 15 invited papers, 33 contributed and 30 poster presentations. It was composed of the seven following sessions:

1. Theoretical aspects of reference systems (Monday 27 August)

2. Reference timescale requirements (Monday 27 August)

3. Topics in celestial mechanics (Monday 27 August)

4. Space mission requirements (Tuesday 28 August)

5. Future requirements for planetary ephemerides (Tuesday 28 August)

6. Relative reference systems (Tuesday 28 August)

7. Concluding discussion and recommendations (Wednesday 29 August)

The Joint Discussion gathered over 100 participants. Presentations covered both completed works and prospects for the future. Session 7 concluded the Joint Discussion with 
a general discussion and recommendations regarding such topics as a redefinition of the astronomical unit, definition and realization of the future ICRS, leap seconds and pulsar time scales. This resulted in the proposal of establishing new Division I working groups; this also resulted in improvements of the wording of the IAU resolution proposal on the re-definition of the astronomical unit, which was submitted to be voted on during the second session of the 28th IAU GA. IAU 2012 Resolution B2 recommends that the astronomical unit be re-defined as a fixed number of Système International d'Unités (SI) metres through a defining constant. For continuity that constant should be the value of the current best estimate in metres as adopted by IAU 2009 Resolution B2 (i.e. $149597870700 \mathrm{~m}$ ).

The JD7 proceedings are composed of the preface, the list of invited, contributed and poster presentations and the summaries of 13 of the invited papers (denoted in the list by paper titles in bold face). Additional Information on the Joint Discussion as well as part of the presentations given during the JD can be found on the JD7 web page at http://www.referencesystems .info/iau-joint-discussion-7.html.

\section{Acknowledgements}

We express our thanks to the SOC for its valuable help in preparing the scientific programme and chairing the sessions, and to all the authors of presentations for their very interesting contributions. We thank the local organizers for the very efficient help during the meeting.

Nicole Capitaine, George Kaplan, Sergei Klioner

JD7 Proceedings co-editors

23 November 2012

\section{List of JD7 invited, contributed and poster presentations}

(Papers titles in bold face denote papers published in these Proceedings.)

Session 1. Theoretical aspects of reference systems

- Relativity in fundamental astronomy: status and prospects by Michael Soffel (invited)

- Celestial dynamics and astrometry in an expanding universe by Sergei Kopeikin (invited)

- Extension of the DSX-formalism to $2 \mathrm{PN}$ order for the problem of light propagation by Chongming Xu, Sergei Klioner, Michael Soffel, Xuejun Wu

- A dynamical reference frame for geophysics and experimental gravitation by Pacôme Delva, Christophe Le Poncin-Lafitte

- Observational evidences for the propagation speed of gravity from Earth tides by Keyun Tang, Wu Wen, Changcai Hua, Shunliang Chi, Qingyu You, Dan-Yu Yu

- Explicit expressions for the global metric and coordinate transformation with local multipole moments by $\mathrm{Yi}$ Xie

- New approach to relativistic celestial reference frames by Olivier Minazzoli

- 2PN light propagation in the scalar-tensor theory: an N-point mass case by Xue-Mei Deng, Yi Xie

- Relativistic spherical multipole moments in astronomy by Jin-He Tao, Wen-Biao Han

- Frequency shift at the post-post-Minkowskian approximation by Christophe Le PoncinLafitte, Aurélien Hees, Stefano Bertone

- Relativistic modeling for high precision space astrometry at post-post Minkowskian approximation by Stefano Bertone, Christophe Le Poncin-Lafitte, Marie-Christine Angonin 
- Transformative relation of kinematical descriptive quantities defined by different spatial referential frame, its property and application by Luo Ji

Session 2. Reference timescale requirements

- A pulsar-based timescale by George Hobbs (invited)

- Long-term stability of atomic time scales by Gérard Petit and Felicitas Arias (invited)

- Perspectives for time and frequency transfer by Philip Tuckey, Pierre Uhrich

- Developments of optical clocks and their comparisons for future time reference by $Y a$ suhiro Koyama, Nobuyasu Shiga, Atsushi Yamaguchi, Tadahiro Goto, Yuko Hanado, Mizuhiko Hosokawa, Miho Fujieda, Hidekazu Hachisu, Tetsuya Ido, Reiko Kojima, Motohiro Kumagai, Ying Li, Kensuke Matsubara, Shigeo Nagano

- Connecting kinematic and dynamic reference frames by D-VLBI by Harald Schuh, Lucia Plank, Matthias Madzak, Johannes Boehm

- Link of reference frames by pulsar observations by Rodin Aleksandr, Sekido Mamoru

- A convention for Coordinated Universal Time by Dennis D. McCarthy

Session 3. Topics in celestial mechanics

- Requirements on space-time reference systems for the BepiColombo and Juno missions by Andrea Milani, Giacomo Tommei, Linda Dimare (invited)

- The trajectory monitoring of spacecraft via VLBI in China's lunar exploration project by Jinling $L i$

- Advanced dynamical models for very well observed asteroids: relativity, non-gravitational effects, perturbations from small bodies by Fabrizio Bernardi, Davide Famocchia, Andrea Milani (invited)

- Phoebe's orbit from ground-based and space-based observations by Josselin Desmars, Shan-Na Li, Radwan Tajeddine, Zheng-Hong Tang

- The geoid computed from a new generalized theory of the figure of the Earth by Cheng- $\mathrm{i}$ Huang, Cheng-Jun Liu, Yu Liu

- May small digital PZT and radio beacons improve the LPhl for future lunar missions? by Jinsong Ping, Xiaoli Su, Hideo Hanada, Alexander Gusev, Jinling Li, Xian Shi, Qinghui Liu

- Progress of astrometric research in Nikolaev Observatory by Anatoliy Ivantsov, Nadia Maigurova, Maxim Martynov, Gennadiy Pinigin

Session 4. Space mission requirements

- Time and frequency transfer with the ESA/CNES ACES-PHARAO mission by Pacôme Delva, Peter Wolf, Frédéric Meynadier, Christophe Le Poncin-Lafitte, Philippe Laurent (invited)

- Celestial reference frame realizations at multiple radio frequency bands by Chris Jacobs (invited)

- Status of Gaia and early operation plans by François Mignard (invited)

- Current status of the Celestial Reference Frame and future prospects by Ralph Gaume

- Next-generation VLBI model: higher accuracy and larger baselines by Sergei Klioner

- New Pulkovo combined catalogues of the radio source positions by Yulia Sokolova, Zinovy Malkin

- Morphology of QSOs; the grid points of the Gaia celestial reference frame by Alexandre Humberto Andrei, Jean Souchay, Roberto Vieira Martins, Sonia Anton, Francois Taris, Sebastien Bouquillon, Marcelo Assafin, Christophe Barache, Julio Ignacio Bueno Camargo, Bruno Coelho, Dario Nepomuceno da Silva Neto

- Systematic effect of the galactic aberration on the ICRS realization and the Earth orientation parameters by Jia-Cheng Liu, Nicole Capitaine, Sébastien Lambert, Zinovy Malkin, Zi Zhu 
- Dipole systematic effect in proper motion of the reference radio sources by Oleg Titov

- Towards ICRF3: preparing the VLBI frame for future synergy with the Gaia frame by Patrick Charlot, Géraldine Bourda

- The Large Quasar Astrometric Catalog V.2, LQAC-2 by Jean Souchay, Alexandre Andrei

- The Gaia reference frame and the acceleration of the solar system barycenter in the presence of quasar variability by Rajesh Kumar Bachchan, David Hobbs, Lennart Lindegren

- Gaia's astrometric global iterative solution: method overview and results by Uwe Lammers, Lennart Lindegren, David Hobbs, William O'Mullane, Ulrich Bastian, Jose Hernandez

- An intensive consideration for the Galactic coordinate system by Zi Zhu, Jia-Cheng Liu

- Reconsidering the International Celestial Reference System based on the effect of the secular aberration by Minghui Xu, Guangli Wang, Ming Zhao

- VLBI application for time and frequency transfer, and comparison with other techniques by Mamoru Sekido, Hiroshi Takiguchi, Thomas Hobiger, Ryuuichi Ichikawa, Miho Fujieda, Tadahiro Goto, Jun Amagai, Kazuhiro Takefuji

- Observations of ERS which are visible in optical domain using $2 \mathrm{~m}$ telescope by Goran Damljanovic, Ivana Milic

- Radio-optical reference frame offsets from CTIO and UCAC4 data by Marion Zacharias, Norbert Zacharias, Charlie T. Finch

- Assessment of stochastic errors of radio source position catalogues by Zinovy Malkin, Julia Sokolova

- Source structure and VLBI position instabilities by Romuald Bouffet, Patrick Charlot, Sébastien Lambert

Session 5. Future requirements for planetary ephemerides

- INPOP: evolution, applications and perspectives by Jacques Laskar, Agnès Fienga, Hervé Manche, Ashok Verma, Mickael Gastineau (invited)

- Linking the planetary ephemerides to the ICRF by William Folkner (invited)

- EPM- the high-precision planetary ephemerides of IAA RAS for scientific research, astronavigation on the Earth and in space by Elena Pitjeva (invited)

- A new approach to asteroid modeling in a planetary ephemeris by Petr Kuchynka

- New developments in spacecraft raw data direct analysis for the INPOP planetary ephemerides by Ashok Verma, Agnès Fienga

- The re-definition of the astronomical unit of length: reasons and consequences by Nicole Capitaine, Sergei Klioner, Dennis McCarthy

- Sensitivity studies of HEO Mars orbiters using perturbation theory by Zhi-Zhou He, Cheng-Li Huang, Mian Zhang

- Global solution of the pulsar clock model and the Earth ecliptic position based on millisecond pulsar timing by Feng Tian, Zheng-Hong Tang

- Asteroids Dynamic Site - AstDyS by Zoran Knezevic, Andrea Milani

Session 6. Relative reference systems

- Connecting terrestrial to celestial reference frames by Zinovy Malkin (invited)

- SOFA, an IAU Service for the future by Catherine Hohenkerk (invited)

- The IERS Conventions (2010): reference systems and new models by Brian Luzum and Gérard Petit (invited)

- Link the EOC4 catalog to the ICRS by Cyril Ron, Jan Vondrák

- A new numerical theory of Earth rotation by Enrico Gerlach, Sergei Klioner, Michael Soffel

- Asymmetric effects in polar motion excitation by Christian Bizouard

- Evaluation of the accuracy of the IAU 2006/2000 precession-nutation by Nicole Capitaine, Sébastien Lambert, Kunliang Yao, Jia-Cheng Liu 
- Impact of IERS conventions (2010) on VLBI-derived reference frames by Robert Heinkelmann, Harald Schuh

- Influence of the inner core on the rotation of the Earth revisited by Alberto Escapa, Jose M. Ferrandiz, Juan Getino

- Researches on predictions of Earth orientation parameters by Xueqing Xu

- A linear operator method to compute the rotational modes of asymmetric 3D Earth by vector spherical harmonics by Mian Zhang, Cheng-Li Huang

- A preliminary study of the relationship between gravity change and plumb line variation on ground in case of anomalous mass underground by Yongzhang Yang, Zhengxin Li, Jinsong Ping

- A study of discovered non-tidal vertical variations in China by Zhengxin Li, Yongzhang Yang

- Excitations of length-of-day variations determined from GPS, SLR and GRACE by Shuanggen Jin, Xinggang Zhang

- IAU SOFA software by Catherine Hohenkerk

- Naval Observatory Vector Astrometry Software (NOVAS) Version 3.1: Fortran, C, and Python Editions by George H. Kaplan, John A. Bangert, Eric G. Barron, Jennifer L. Bartlett, Wendy Puatua, William Harris, Paul Barrett

- Nutation determination using the Global Positioning System by Kunliang Yao, Nicole Capitaine, Jean-Yves Richard

- Progress in SLR - GPS co-location at San Juan (Argentina) station by Hernan Alvis Rojas, Ana Pacheco, Ricardo Podestá, Eloy Actis, Jinzeng Li, Zhiqiang Yin, Rui Wang, Dongping Huang, Raùl Márquez, Sonia Adarvez, Johana Quinteros, Pablo Cobos, Andrés Aracena

- Russian Astronomical Software by Marina V. Lukashova, Nina I. Glebova, Ilja N. Netsvetaev, Galina A. Netsvetaeva, Ekaterina Ju. Parijskaja, Elena V. Pitieva, Michael L. Sveshnikov, Vladimir I. Skripnichenko

- SLR and GPS spatial techniques in ITRF. Argentine results by Eloy Vicente Actis, Ricardo Podestá, Ana M. Pacheco, Hernán Alvis Rojas, Zhiqiang Yin, Jinzeng Li, Yanben Han, Weidong Liu, Rui Wang, Dongping Huang, Raùl Márquez, Sonia Adarvez, Matias Flores, Diego Brizuela, Jesica Nievas

- The geopotential computed from global crustal models by Cheng-Jun Liu

- The interior structure of the Earth constrained from gravity field data and the generalized theory of the figure of the Earth by Cheng-Jun Liu, Cheng-Li Huang

- The new expansion of annual aberration into trigonometric series by Cyril Ron, Jan Vondrák

- New role of astrometry technique in sciences and technologies by Zhengxin $L i$ 